\title{
Discursos honestos e mentirosos: Há como discriminá-los pela voz?
}

\author{
Honest and lying speeches: Is it possible to discriminate them by voice? \\ Discursos honestos y mentirosos: ¿Hay cómo discriminarlos por la voz?
}

\author{
Nathália Monteiro Santos \\ ORCID: https://orcid.org/0000-0003-2746-5361 \\ Universidade Federal de Sergipe, Brasil \\ E-mail: nathaliamonteiro.fono@gmail.com \\ Josefa Aparecida Ribeiro Bispo \\ ORCID: https://orcid.org/0000-0001-7553-2122 \\ Universidade Federal de Sergipe, Brasil \\ E-mail: aparecidaribeiro17@gmail.com \\ Mariza de Jesus Santos \\ ORCID: https://orcid.org/0000-0002-8394-9996 \\ Universidade Federal de Sergipe, Brasil \\ E-mail: marizasantostb@hotmail.com \\ José Marcos de Jesus Santos \\ ORCID: https://orcid.org/0000-0001-5122-1469 \\ Universidade de São Paulo, Brasil \\ E-mail: jsmarcos@usp.br \\ Ariane Damasceno Pellicani \\ ORCID: https://orcid.org/0000-0003-0390-9175 \\ Universidade Federal de Sergipe, Brasil \\ E-mail: ariane.pellicani.ap@gmail.com \\ Carla Patrícia Hernandez Alves Ribeiro César \\ ORCID: https://orcid.org/0000-0002-9439-9352 \\ Universidade Federal de Sergipe, Brasil \\ E-mail: carlacesar@globo.com
}

\begin{abstract}
Resumo
Objetivo: Comparar parâmetros vocais entre discursos honestos e falsos. Método: 40 sujeitos (20 de cada gênero), entre 19 e 58 anos $(28,82 \pm 9,68)$, foram submetidos a testes de discurso honestos e/ou falsos. Para a análise vocal foi solicitada a emissão sustentada da vogal /e/ em frequência e intensidade habitual previamente às situações pré-teste e teste. As amostras de voz e fala foram capturadas por meio de microfone e vídeo. Os parâmetros vocais analisados foram frequência fundamental (f0), pitch, loudness, tensão fonatória, instabilidade e ataque vocal. Resultados: os parâmetros vocais que apresentaram diferenças estatisticamente significantes foram a tensão $(p=0,004)$ e o ataque vocal $(\mathrm{p}=0,01)$. A loudness apresentou uma tendência a aumento no discurso falso ( $\mathrm{p}=0,08)$. Conclusão: os parâmetros vocais de tensão e ataque vocal se diferenciaram entre os discursos falsos e honestos, o que podem sugerir uma possível mudança no comportamento vocal frente a discursos mentirosos.
\end{abstract}

Palavras-chave: Voz; Fonoaudiologia; Decepção.

\begin{abstract}
Objectives: to compare vocal parameteres between honest and lying speech. Methods: 40 subjects (20 men and 20 women), with ages between 19 and 58 years, were submitted to tests of honest and lying speeches. To acoustic analyses, the sustainted vowel /ae/ with habitual frequency and intensity of voice were requested before the test and in the test. Voice and speech samples were captured by microfone and vídeo records The vocal parameteres analysed were fundamental frequency (f0), pitch, loudness, phonatory tension, instability and vocal attack. Results: the vocal parameteres of tension $(p=0,004)$ and vocal attack $(p=0,01)$ demonstrated estatistically significant diferences. The loudness demonstrated a tendency to increase in lying speech $(\mathrm{p}=0,08)$. Conclusion: the vocal parameteres of tension and vocal attack seems to differenciate between honest and lying speech, which may suggest a possible change in vocal behavior on lying speeches.
\end{abstract}

Keywords: Voice; Speech language and hearing sciences; Deception.

\section{Resumen}

Objetivo: Comparar parámetros vocales entre discursos honestos y falsos. Método: 40 sujetos (20 de cada género), entre 19 y 58 años $(28.82 \pm 9.68)$, se sometieron a pruebas de habla honestas y / o falsas. Para el análisis vocal, se solicitó la emisión sostenida de la vocal / e / en frecuencia e intensidad habitual antes de las situaciones de prueba previa y prueba. Las muestras de voz y habla fueron capturadas usando un micrófono y video. Los parámetros vocales 
analizados fueron frecuencia fundamental (f0), tono, volumen, tensión fonatoria, inestabilidad y ataque vocal. Resultados: los parámetros vocales que mostraron diferencias estadísticamente significativas fueron la tensión $(\mathrm{p}=$ $0.004)$ y el ataque vocal $(p=0.01)$. La sonoridad tiende a aumentar en el habla falsa $(\mathrm{p}=0.08)$. Conclusión: los parámetros vocales de tensión y ataque vocal diferían entre discursos falsos y honestos, lo que puede sugerir un posible cambio en el comportamiento vocal cuando se enfrentan a discursos falsos.

Palabras clave: Voz; Fonoaudiología; Decepción.

\section{Introdução}

As emoções podem ser reconhecidas por meio dos recursos vocais, sendo que as vozes com conteúdo emocional com maior facilidade para o reconhecimento são a raiva, a tristeza e o medo, enquanto a surpresa e a aversão são tidas como mais difíceis neste sentido (Paixão et al., 2010). Reconhecer as características emocionais vocais auxilia na compreensão da mensagem, sendo um instrumento importante para a comunicação emocional (Paixão et al., 2010), adquiridas pela interação humana.

Da mesma forma, a mentira também é aprendida, levando-se em consideração que para tanto, faz-se necessário observação, prática, capacidades cognitivas e de raciocínio desenvolvidas (Manen \& Levering, 1996). Sua detecção tem sido alvo de pesquisas nacionais e internacionais (Lo \& Tseng, 2018; Trifiletti et al., 2020) que investigam respostas pupilares ou marcadores eletrofisiológicos durante discursos falsos, porém poucos estudos têm focado sobre as características vocais no ato do mentir.

Durante a mentira pode haver a manifestação dos chamados "vazamentos", ou seja, sinais inconscientes que podem indicar que o sujeito esteja mentindo (Fexeus, 2015), sendo que o senso comum indica que pode haver tensão vocal, uma vez que raramente a voz transmite mensagens emocionais falsas (Ekman, 2011; Masip et al., 2004). Pode haver, nos discursos desonestos, a produção de uma voz mais aguda (Ekman, 2011; Freitas-Magalhães, 2017), assincronia da entonação vocal com o contexto/conteúdo da mensagem (Freitas-Magalhães, 2008), modificação na loudness (Fexeus, 2015; Freitas-Magalhães, 2017) ou ainda mudanças dos parâmetros vocais como frequência e velocidade sem motivo plausível (Fexeus, 2015). Porém, tais manifestações foram atribuídas a discursos falsos por meio de inferência, sem a realização de estudos observacionais ou análise de tais parâmetros por especialistas da área, com exceção de duas, uma realizada em 1994 (Cestaro \& Dollins, 1994) e outra em 2002 (Suzuki et al., 2002), em que não encontraram alterações vocais durante a mentira.

Desta forma, justifica-se a realização desta pesquisa a fim de elucidar se a voz pode trazer indícios de que uma pessoa esteja ou não mentindo. Sendo assim, o objetivo do presente estudo foi comparar parâmetros vocais entre discursos honestos e falsos.

\section{Metodologia}

\section{Desenho do estudo e aspectos éticos}

Trata-se de um estudo com abordagem quantitativa (A. Pereira et al., 2018), transversal, aprovado pelo Comitê de Ética em Pesquisa com número CAEE 42025115.9.0000.5546, sendo parte da investigação "Detecção de mentira por meio dos sinais das microexpressões faciais, verbais e vocais".

\section{Amostra}

Foram convidados a participar do estudo indivíduos dos gêneros feminino e masculino, autodeclarados saudáveis, com adequada compreensão e expressão oral e que não apresentassem sinais e/ou cicatrizes na face. Não foram aceitos indivíduos com queixa vocal, auditiva, alterações neurológicas, psiquiátricas, visual, usuários de substâncias psicoativas, e aqueles que tivessem feito uso de bebidas alcoólicas no dia anterior a coleta de dados. 
Desta forma, a amostra foi constituída por 40 sujeitos (amostra de conveniência), distribuídos igualitariamente entre os gêneros, com idades entre 19 e 58 anos (média 28,82 \pm 9,68). Quanto ao grau de escolaridade, 15\% apresentaram o ensino fundamental completo, $20 \%$ o médio e $65 \%$, o superior (completo ou em fase de conclusão). A todos os participantes foi explicado oralmente a proposta da pesquisa e ao concordarem, receberam uma carta explicativa e assinaram os Termos de Consentimento Livre e Esclarecido.

\section{Procedimentos}

\section{Discursos honesto e falsos}

Inicialmente, foi realizada a fase pré-teste por meio da coleta de amostras de fala espontânea, no qual era solicitado ao indivíduo dizer sua idade, se estudava, curso e motivo que o levou a estudar o referido curso. Nesta fase, todos os participantes eram orientados a dizer a verdade, ou seja, o discurso era tido como honesto.

De forma aleatória, o participante recebeu uma ficha contendo uma das duas frases escritas em um papel: "FALE A VERDADE" ou "MINTA", entregue ao participante sob a forma de sorteio. Baseado em um estudo (M. E. Pereira et al., 2006), solicitou-se ao participante para que dissesse o nome de seus pais, irmãos, bairro onde mora, o nome de uma pessoa que apreciava e o motivo de gostar dessa pessoa.

De forma a aumentar a motivação para a emissão de um discurso falso, os acadêmicos receberam a orientação verbal, antecipadamente, de que em muitas ocasiões, tanto na vida cotidiana quanto na profissional, para alcançarem sucesso precisariam esconder o medo, a aflição, o nojo ou ainda, usar de um discurso mentiroso para terem a cooperação dos outros ou para conseguirem se portar de acordo com o desejado (Honorio, 2012).

Assim, na fase teste, metade dos participantes produziu discurso honesto e a outra, falso, selecionados de forma aleatória, conforme ilustrado na Figura 1 (Fluxograma da pesquisa).

Figura 1. Fluxograma da pesquisa.

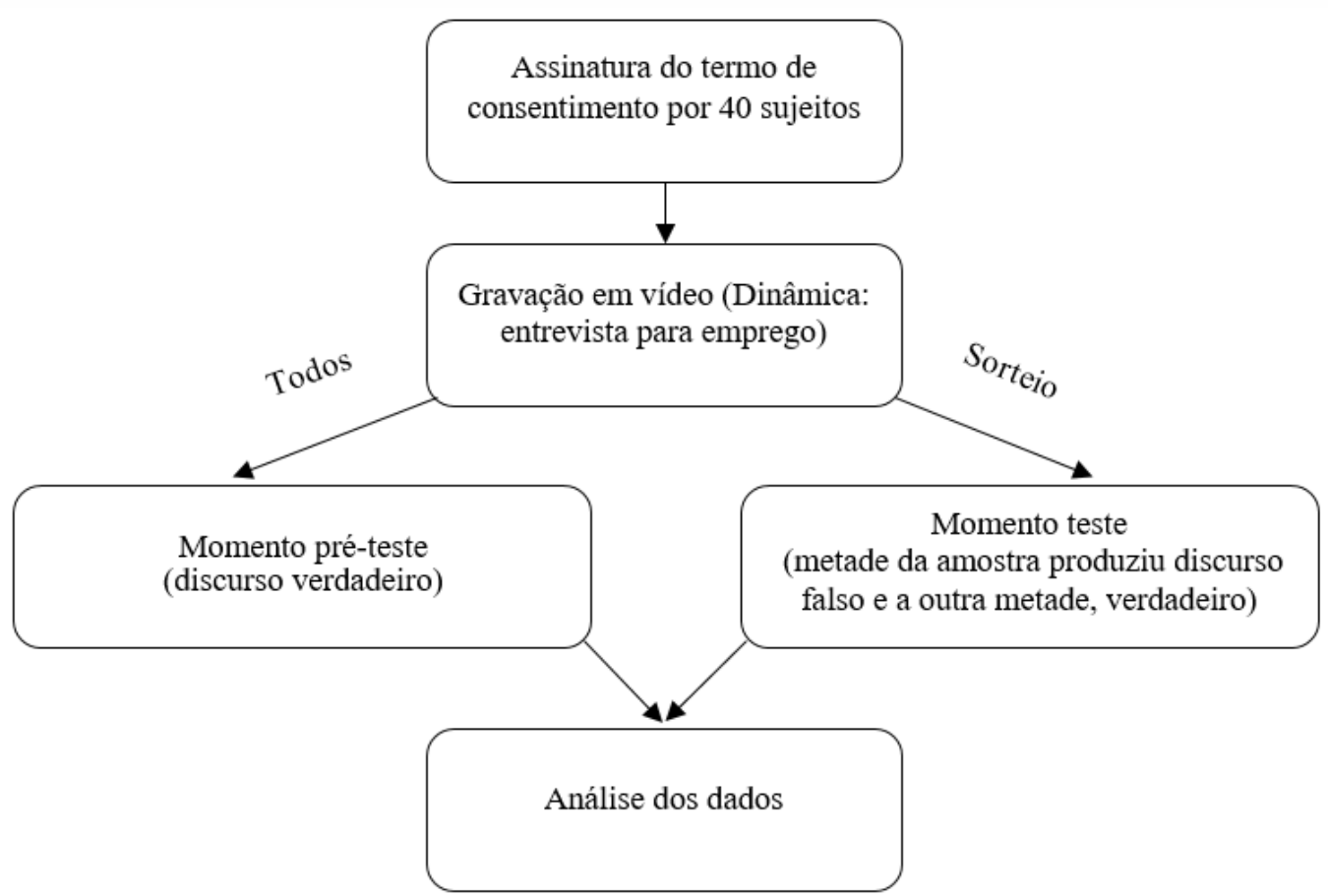

Fonte: Autores (2021). 


\section{Captura das amostras vocais}

Todas as produções vocais das amostras de fala em discurso honesto e falso foram registradas por captura de vídeo e áudio.

Nos momentos anteriores ao pré-teste e teste foi solicitado aos participantes a emissão da vogal /e/ sustentada no tempo máximo de fonação, com voz em intensidade e frequência habitual. Para a captura das amostras em áudio da voz e fala foi utilizado um microfone sensível unidirecional com fio, da marca Shure (modelo SM58), distante da boca do participante a $3 \mathrm{~cm}$, em ângulo de $90^{\circ}$, em pedestal, acoplado a uma interface de áudio externa da marca Behringer U-PHORIA UM2 que estava conectado diretamente a um notebook da marca Dell com processador Intel® CoreTM i5. As amostras de voz e fala foram registradas por meio do programa Audacity®, com frequência de amostragem de 44.100Hz, 16 bits e mono canal.

Para a gravação de vídeo foi utilizada uma máquina fotográfica na função de vídeo, da marca Sony Cyber Shot, digital (7,2 megapixels), modelo DSC P200. Para tanto, a máquina foi colocada sobre um tripé (para evitar alterações decorrentes de má postura do fotógrafo), ajustada a uma altura de um metro do solo e à distância de dois metros de um fundo constante

Todas as amostras de voz foram registradas individualmente, em ambiente silencioso. Os participantes permaneceram sentados, de forma confortável, em uma cadeira, com pernas flexionadas em $90^{\circ}$ e pés no chão.

\section{Análise}

Para a extração do parâmetro acústico da frequência fundamental foi utilizado o software Praat (versão 4.6.10) (Boersma \& Weenick, 2006), no qual foram excluídos o início e final da emissão visando o trecho de maior estabilidade da amostra vocal.

Por meio das amostras de voz e vídeo dos discursos falsos e honestos foram analisados os parâmetros de pitch (adequado ou inadequado e se houve modificação entre os discursos - P), a loudness (adequada ou inadequada e se houve modificação entre os discursos - L), a tensão (presença ou não na região laríngea e/ou cervical - T), a instabilidade vocal (presença ou não) e o ataque vocal (isocrônico - AVI, soproso - AVS ou brusco - AVB).

Os registros de voz e vídeo (extensão do arquivo “.MOV”) foram apresentadas a quatro avaliadores pelo método direto, ou seja, sabiam que o tema da pesquisa versava sobre a mentira e, de forma independente, anotaram suas observações em uma ficha criada para tal finalidade. Os resultados foram posteriormente discutidos em conjunto e as discrepâncias foram sanadas por consenso entre os avaliadores.

Os dados foram tabulados no Microsoft Office Excel (2010) e importados para o software IBM® SPSS - Statistical Package for the Social Sciences, versão 2.0 para Windows, no qual foram analisados.

Para a análise estatística foram utilizadas as técnicas uni e bivariada para obtenção da distribuição dos valores de frequência e de porcentagem. Ao considerar o tipo de dados (paramétricos) e o tipo de variáveis (qualitativa ordinal e nominal), utilizou-se o Qui-quadrado de independência de Pearson para obter o valor de "p" entre as associações e também o Exato de Fisher para as tabelas cruzadas 2x2 com valores esperados menores que cinco, sendo estatisticamente significativa, em ambos os casos, às que apresentaram $\mathrm{p}<0,05$. O Coeficiente de Pearson também foi usado para mensurar a intensidade das correlações.

A intensidade das correlações foi avaliada de acordo com os seguintes parâmetros: 0,00 a 0,29 (correlação inexistente), 0,30 a 0,49 (correlação baixa), 0,50 a 0,69 (correlação moderada), 0,70 a 0,89 (correlação elevada) e 0,90 a 1,00 (correlação muito elevada), seja com direção positiva $(>0,00)$ ou negativa $(<0,00)$.

A análise da frequência fundamental foi analisada em função do gênero do participante para os discursos honestos e falsos. Nesta avaliação foi utilizado o Teste t de Student pareado para analisar se a diferença observada entre as médias das frequências da voz era estatisticamente significativa $(\mathrm{p}<0,05)$. 
Dessa forma, as variáveis relacionadas às características vocais (pitch, loudness, tensão, instabilidade e ataque vocal) e a frequência fundamental foram analisadas segundo o tipo de discurso (honesto e falso).

\section{Resultados}

Os quarenta sujeitos elegíveis para participar deste estudo foram analisados sem perdas ou desistências durante a pesquisa.

A média de idade dos participantes do grupo do discurso honesto foi de 28,9 anos (idades entre 20 e 58 anos, desvio padrão=9,7) e do discurso falso de 28,7 anos (idades entre 19 e 57 anos, desvio padrão=9,8). Houve distribuição igualitária entre os sexos (50\% masculino e 50\% feminino em cada grupo) e também entre o nível de escolaridade.

Os resultados da avaliação perceptivo-auditiva demonstraram que os parâmetros que diferiram entre os discursos honestos e falsos foram a tensão e o ataque vocal (soproso ou brusco), com relevância estatisticamente significante (Tabela 1).

Tabela 1 - Características vocais comparadas entre os grupos cujos discursos correspondiam à verdade e à mentira, nos momentos pré-teste e teste.

\begin{tabular}{|c|c|c|c|c|c|c|c|}
\hline \multirow[t]{2}{*}{ Características vocais } & \multicolumn{2}{|c|}{$\begin{array}{c}\text { Grupo do discurso } \\
\text { honesto } \\
(\mathbf{n}=\mathbf{2 0})^{*}\end{array}$} & \multicolumn{2}{|c|}{$\begin{array}{c}\text { Grupo do discurso } \\
\text { falso } \\
(\mathbf{n}=\mathbf{2 0})^{*}\end{array}$} & \multicolumn{3}{|c|}{ Testes } \\
\hline & $\mathbf{N}$ & $\%$ & $\mathbf{N}$ & $\%$ & $\mathbf{p}^{*}$ & OR & IC $95 \%$ \\
\hline \multicolumn{8}{|l|}{ Pitch } \\
\hline Mantido & 17 & 85 & 17 & 85 & \multirow{2}{*}{$0,449^{\mathrm{a}}$} & \multirow{2}{*}{0,33} & \multirow{2}{*}{$0,06-1,65$} \\
\hline Modificado & 3 & 15 & 3 & 15 & & & \\
\hline \multicolumn{8}{|l|}{ Loudness } \\
\hline Mantido & 17 & 85 & 16 & 80 & \multirow{2}{*}{$0,088^{\mathrm{a}}$} & \multirow{2}{*}{15,0} & \multirow{2}{*}{$0,89-25,1$} \\
\hline Modificado & 3 & 15 & 4 & 20 & & & \\
\hline \multicolumn{8}{|l|}{ Tensão } \\
\hline Presente & 7 & 35 & 8 & 40 & \multirow{2}{*}{$0,004^{a}$} & \multirow{2}{*}{33,0} & \multirow{2}{*}{$2,45-44,3$} \\
\hline Ausente & 13 & 65 & 12 & 60 & & & \\
\hline \multicolumn{8}{|l|}{ Instabilidade } \\
\hline Presente & 6 & 30 & 6 & 30 & \multirow{2}{*}{$0,432^{\mathrm{a}}$} & \multirow{2}{*}{0,45} & \multirow{2}{*}{$0,03-1,98$} \\
\hline Ausente & 14 & 70 & 14 & 70 & & & \\
\hline \multicolumn{8}{|l|}{ Ataque vocal } \\
\hline Equilibrado & 7 & 35 & 5 & 25 & \multirow{2}{*}{$\mathbf{0 , 0 1 5 ^ { \mathrm { b } }}$} & \multirow{2}{*}{16,0} & \multirow{2}{*}{$1,27-20,0$} \\
\hline Alterado & 13 & 65 & 15 & 75 & & & \\
\hline
\end{tabular}

Legenda: ${ }^{\mathrm{a} E x a t o}$ de Fisher, ${ }^{\mathrm{b}} \mathrm{Qui}-q u a d r a d o, ~{ }^{\mathrm{c}}$ Teste t pareado, OR= Razão de Chances, IC 95\%= Intervalo de Confiança de $95 \%$.

Foi empregado negrito nas associações estatisticamente significativas. $* \mathrm{p}<0,05$

Fonte: Autores.

A frequência fundamental (f0) não revelou diferenças estatisticamente significantes entre os discursos honesto e verdadeiro (Tabela 2). 
Tabela 2. Comparação da média das frequências fundamentais dos participantes e dos discursos honesto e falso.

\begin{tabular}{lccc}
\hline Gênero/Discurso & Média da f0 no discurso honesto & Média da f0 no discurso honesto & p-valor* \\
Gênero Feminino & $207,6 \mathrm{~Hz}$ & $211,64 \mathrm{~Hz}$ & 0,697 \\
Gênero Masculino & $139,3 \mathrm{~Hz}$ & $131,8 \mathrm{~Hz}$ & 0,452 \\
Total & $173,45 \mathrm{~Hz}$ & $171,72 \mathrm{~Hz}$ & 0,195 \\
\hline
\end{tabular}

Legenda: $\mathrm{Hz}=$ Hertz. $*$ Teste $\mathrm{t}$ de Student pareado.

$* * p<0,05$

Fonte: Autores.

\section{Discussão}

O som gerado pela vibração das pregas vocais, a voz, possibilita ao ser humano expressar reflexões, opiniões, pensamentos e desempenha função importante na comunicação e no cotidiano (Z. Zhang, 2016). Além disso, por meio da voz é possível disfarçar a identidade, falsificando-a em diversas situações para esconder a verdade (C. Zhang \& Tan, 2008). A literatura vem abordando técnicas e estudos a respeito da detecção de incongruências na fala e no corpo, porém poucos estudos têm verificado se há ou não pistas vocais que possam oferecer indícios de que a pessoa esteja mentindo, justificando a realização desta pesquisa.

Durante o discurso, podem ser observados alguns sinais de mentira na comunicação verbal como aumento na velocidade da fala, mudança no tom da voz entre outros aspectos (Quinta \& Coelho, 2009).

No presente estudo notou-se que, apesar do aumento absoluto na loudness e modificação na frequência fundamental da voz (aumento para as mulheres e diminuição para os homens), não houve mudança estatisticamente significante entre os tipos de discurso para estes parâmetros vocais. Em um estudo (Kirchhübel \& Howard, 2013) também observaram discretas diferenças vocais acústicas nos números absolutos, porém sem resultado estatístico significante, ratificando as evidências obtidas. Entretanto, outros pesquisadores (Jürgens et al., 2011) demonstraram que na análise vocal de atores comparadas com vozes espontâneas, houve variabilidade da frequência fundamental e na qualidade vocal, de forma sutil (Jürgens et al., 2011).

A tensão fonatória apresentou diferença estatística no presente estudo, no qual esteve presente na maioria dos discursos falsos (mentirosos). De acordo com a literatura, a tentativa de controle comportamental durante a mentira pode resultar em tensão muscular (Freitas-Magalhães, 2017; Memon et al., 2003) frente à ansiedade da situação (Freitas-Magalhães, 2013, 2017), porém os autores não especificaram os locais corpóreos de tensão.

A presença de tensão e retração muscular cervical está associada às alterações vocais e cervicais, comprometendo o trato vocal e a região musculoesquelética cervical (Menoncin et al., 2010), inferindo-se que possa haver modificações na qualidade vocal durante o ato de mentir. Isto porque a musculatura extrínseca laríngea participa da produção vocal auxiliando a musculatura intrínseca da laringe a executar suas funções (Peter et al., 2001). Cabe ainda ressaltar que há resultados divergentes na literatura sobre a presença de estresse vocal na mentira, tendo em vista que tal estresse não precisa, necessariamente, estar atrelado à mentira (Harnsberger et al., 2009).

Além disso, percebe-se que a mudança do ataque vocal soproso para o brusco em que há maior e mais forte contato entre as pregas vocais pode ter sofrido influência também do nervosismo e da tensão corporal durante a mentira. O ataque vocal, ou seja, o modo como a fonação é iniciada, pode ser isocrônico, brusco ou soproso. Neste estudo observou-se que houve mudança do ataque vocal soproso, em que há maior escape de ar devido ao fechamento glótico incompleto, para o ataque vocal brusco que, segundo a literatura (Behlau et al., 2001), pode estar relacionado à hipertonia da musculatura.

Nota-se na literatura a necessidade de mais estudos sobre a detecção da mentira a respeito das características vocais com participação do fonoaudiólogo, sendo este o profissional apto para esta função, com o propósito de mais achados a respeito da relação entre esses fatores: voz e detecção de mentira, o que pode auxiliar na área de perícia e voz forense. 
A limitação deste estudo compreendeu na dificuldade da proposição do método, uma vez que não foram encontrados estudos com o mesmo objetivo, dificultando a comparação dos resultados. Assim, incitam-se mais pesquisas na área a fim de ratificar ou não as evidências obtidas.

\section{Conclusão}

Os parâmetros vocais de tensão e ataque vocal se diferenciaram entre os discursos falsos e honestos, o que podem sugerir uma possível mudança no comportamento vocal frente a discursos falsos (mentirosos). Por se tratar de parâmetros e análises da expertise do fonoaudiólogo, cabe a esse profissional a realização de mais pesquisas para uma melhor condução da aplicabilidade da avaliação fonoaudiológica frente a análise dos discursos e detecção da mentira por meio da voz e fala. Sugere-se para pesquisas futuras um maior tamanho amostral com realização de exames laríngeos nos participantes, além da identificação da mentira por ouvintes leigos e fonoaudiólogos, apenas por áudio, para verificar se os recursos vocais permitem essa diferenciação.

\section{Agradecimentos}

Ao apoio financeiro do Conselho Nacional de Desenvolvimento Científico e Tecnológico (CNPq).

\section{Referências}

Behlau, M., Madazio, G., Feijó, D., \& Pontes, P. (2001). Avaliação de voz. In Voz - o livro do especialista, volume 1 (pp. 85-245). Revinter.

Boersma, P., \& Weenick, D. (2006). Praat manual. Amsterdam: University of Amsterdam. http://www.fon.hum.uva.nl/praat/.

Cestaro, V. L., \& Dollins, A. B. (1994). An analysis of voice responses for the detection of deception. Department of Defense Polygraph Institute.

Ekman, P. (2011). A linguagem das emoções: revolucione sua comunicação e seus relacionamentos reconhecendo todas as expressões das pessoas ao redor. Lua de Papel.

Fexeus, H. (2015). A arte de ler mentes: como interpretar gestos e influenciar pessoas sem que elas percebam. (5th ed.). Vozes.

Freitas-Magalhães, A. (2008). O código de Ekman: o cérebro, a face e a emoção. Leya.

Freitas-Magalhães, A. (2013). A psicologia das emoções - o fascínio do rosto humano. Leya.

Freitas-Magalhães, A. (2017). Por que me mentes? Ensaio sobre a face da mentira. Leya.

Harnsberger, J. D., Hollien, H., Martin, C. A., \& Hollien, K. A. (2009). Stress and Deception in Speech: Evaluating Layered Voice Analysis. Journal of Forensic Sciences, 54(3), 642-650. https://doi.org/10.1111/j.1556-4029.2009.01026.x

Honorio, F. F. (2012). Precisão na detecção de mentiras: investigação sobre o efeito da detecção indireta. Universidade de Brasília.

Jürgens, R., Hammerschmidt, K., \& Fischer, J. (2011). Authentic and Play-Acted Vocal Emotion Expressions Reveal Acoustic Differences. Frontiers in Psychology, 2. https://doi.org/10.3389/fpsyg.2011.00180

Kirchhübel, C., \& Howard, D. M. (2013). Detecting suspicious behaviour using speech: Acoustic correlates of deceptive speech - An exploratory investigation. Applied Ergonomics, 44(5), 694-702. https://doi.org/10.1016/j.apergo.2012.04.016

Lo, Y.-H., \& Tseng, P. (2018). Electrophysiological markers of working memory usage as an index for truth-based lies. Cognitive, Affective, \& Behavioral Neuroscience, 18(6), 1089-1104. https://doi.org/10.3758/s13415-018-0624-2

Manen, M. Van, \& Levering, B. (1996). O Segredo na Infância: intimidade privacidade e o self reconsiderado. Instituto Piaget.

Masip, J., Garrido, E., \& Herrero, C. (2004). La detección de la mentira mediante la medida de la tensión en la voz: una revisión crítica. Estudios de Psicología, 25(1), 13-30. https://doi.org/10.1174/021093904773486980

Memon, A., Vrij, A., \& Bull, R. (2003). Psychology and law: truthfulness, accuracy and credibility (2nd ed.). Wiley.

Menoncin, L. C. M., Jurkiewicz, A. L., Silvério, K. C. A., Camargo, P. M., \& Wolff, N. M. M. (2010). Alterações musculares e esqueléticas cervicais em mulheres disfônicas. Arquivos Internacionais de Otorrinolaringologia (Impresso), 14(4), 461-466. https://doi.org/10.1590/S1809-48722010000400014

Paixão, R., Coelho, L., \& Ferreira, J. (2010). Teste de Reconhecimento Paralinguístico das Emoções. Psychologica, 53, 423-446. https://doi.org/10.14195/1647-8606_53_21 
Research, Society and Development, v. 10, n. 9, e46610918266, 2021

(CC BY 4.0) | ISSN 2525-3409 | DOI: http://dx.doi.org/10.33448/rsd-v10i9.18266

Pereira, A., Shitsuka, D., Parreira, F., \& Shitsuka, R. (2018). Metodologia da pesquisa científica (1st ed.). Núcleo de Tecnologia Educacional da Universidade Federal de Santa Maria.

Pereira, M. E., Brasileiro, R., Silva, J. F. da, Silva, P. B. e, Brachi, D., \& Albuquerque, F. (2006). Estereótipos, mentiras e videotape: estudos experimentais sobre a acurácia na identificação da mentira. Psicologia Em Estudo, 11(1), 209-218. https://doi.org/10.1590/S1413-73722006000100024

Peter, G., Pinho, S., \& Assencio-Ferreira, V. (2001). Musculatura extrínseca da laringe e sua participação na produção vocal. CEFAC, $3,165-173$.

Quinta, N. C. de C., \& Coelho, C. (2009). Contando e detectando mentiras: efeito do feedback sobre o desempenho. Psicologia: Teoria e Pesquisa, 25(1), 137-145. https://doi.org/10.1590/S0102-37722009000100016

Suzuki, A., Watanabe, S., Taheno, Y., Kosugi, T., Kasuya, T., \& Senter, D. (2002). Possibility of detecting deception by voice analysis. Polygraph, 31, 129134.

Trifiletti, E., D’Ascenzo, S., Lugli, L., Cocco, V. M., Di Bernardo, G. A., Iani, C., Rubichi, S., Nicoletti, R., \& Vezzali, L. (2020). Truth and lies in your eyes: Pupil dilation of White participants in truthful and deceptive responses to White and Black partners. PLOS ONE, 15(10), e0239512. https://doi.org/10.1371/journal.pone.0239512

Zhang, C., \& Tan, T. (2008). Voice disguise and automatic speaker recognition. Forensic Science International, 175(2-3), 118-122. https://doi.org/10.1016/j.forsciint.2007.05.019

Zhang, Z. (2016). Mechanics of human voice production and control. The Journal of the Acoustical Society of America, 140(4), 2614-2635. https://doi.org/10.1121/1.4964509 